\title{
Novel mutation of the PRRT2 gene in two cases of paroxysmal kinesigenic dyskinesia: Two case reports
}

\author{
JIAJIA FANG ${ }^{1}$, SHIGE WANG ${ }^{2}$, GUOHUA ZHAO ${ }^{1,3^{*}}$ and LICAO $^{2 *}$ \\ ${ }^{1}$ Department of Neurology, The Fourth Affiliated Hospital, Zhejiang University School of Medicine, Yiwu, Zhejiang 322000; \\ ${ }^{2}$ Department of Neurology and Institute of Neurology, Ruijin Hospital Affiliated to \\ Shanghai JiaoTong University School of Medicine, Shanghai 200025; \\ ${ }^{3}$ Department of Neurology, The Second Affiliated Hospital, \\ Zhejiang University School of Medicine, \\ Hangzhou, Zhejiang 310009, P.R. China
}

Received October 14, 2019; Accepted February 25, 2020

DOI: $10.3892 /$ br.2020.1293

\begin{abstract}
Paroxysmal kinesigenic dyskinesia (PKD) is a rare condition characterized by recurrent brief episodes of dystonia, chorea, athetosis or any combination of these, without alterations of consciousness. The proline-rich transmembrane protein 2 (PRRT2) gene has been widely investigated as a causative gene of PKD. To date, a cluster of pathogenic variants associated with PKD have been identified in the PRRT2 gene. In the present case report, two Chinese patients with sporadic PKD are discussed. Genetic analysis revealed a de novo heterozygous missense mutation, c.955G $>$ T (p.Val319Leu) in exon 3 of the PRRT2 gene. Compared with the commonly reported clinical manifestation of PRRT2-associated PKD, the patients in this report showed several primary distinctive features. The mutations identified in the present analysis expand upon the mutation spectrum of the PRRT2 gene, and this newly found variant further reinforces the importance of the PRR 2 gene in PKD.
\end{abstract}

\section{Introduction}

Paroxysmal kinesigenic dyskinesia (PKD) is a rare condition characterized by recurrent brief episodes of dystonia, chorea,

Correspondence to: Dr Li Cao, Department of Neurology and Institute of Neurology, Ruijin Hospital Affiliated to Shanghai JiaoTong University School of Medicine, 197 Ruijin Second Road, Shanghai 200025, P.R. China

E-mail: caoli@rjlab.cn

Dr Guohua Zhao, Department of Neurology, The Fourth Affiliated Hospital, Zhejiang University School of Medicine, 1 Shangcheng Avenue, Yiwu, Zhejiang 322000, P.R. China

E-mail: gzhao@zju.edu.cn

${ }^{*}$ Contributed equally

Key words: paroxysmal kinesigenic dyskinesia, proline-rich transmembrane protein 2, mutation athetosis or a combination thereof, without alteration of consciousness. PKD may be considered as a pure form, where patients only present with dystonia, chorea and athetosis, or as a complicated form, were patients present with PKD combined with benign familial infantile convulsions or hemiplegic migraine (1). Episodes are usually triggered by sudden voluntary movements (1). The majority of PKD cases are inherited with an autosomal dominant pattern with complete or incomplete penetrance; although there are reported sporadic (1). In 2011, Chen et al (2) first reported mutations in the gene encoding proline-rich transmembrane protein 2 (PRRT2, chromosome 16p11.2) as the genetic cause of PKD in eight families (2). At present, mutations of the PRRT2 gene are the major cause of PKD, with a frequency ranging from $40 \%$ to $>90 \%$, depending on case ascertainment $(1,3)$. The frameshift mutation c.649dupC (p.Arg217Profs*8), which results in the presence of a premature stop codon, is a hotspot mutation (4). In the present report, two cases of sporadic PKD with sequencing analysis of the PRRT2 gene are described, identifying a potentially novel heterozygous missense mutation, c.955G >T (p.Val319Leu), in exon 3 of the PRR2 gene.

\section{Case reports}

Clinical presentation. Case 1: The index patient, II:2, in Family A (Fig. 1A), an 18-year-old male, was referred to the neurology clinic (The Fourth Affiliated Hospital, Zhejiang University School of Medicine), due to a 3-year history of paroxysmal left limb dystonia. The episodes were triggered by suddenly rising from sitting, and occasionally, being startled, and were characterized by brief episodes of the left limbs rolling outward involuntarily, with the wrists and ankles bent transiently, head turning and slightly impaired speech with preserved consciousness. The episodes usually lasted 5-10 sec with a frequency of up to 10-20 episodes per a day. Epilepsy was suspected initially, and $200 \mathrm{mg}$ per day sodium valproate and $100 \mathrm{mg}$ per day Dilantin were prescribed. The number of episodes were reduced, but continued to occur 3-5 times daily. Neurological examination did not show any abnormalities. The patient had no adverse perinatal events and had developed 
normally. The $24 \mathrm{~h}$ video electroencephalography (VEEG) and brain magnetic resonance imaging (MRI) were normal, and laboratory examinations, including complete cell count, liver function tests, renal function tests, blood sugar measurement and thyroid function were normal. PKD was diagnosed based on the diagnostic criteria (5) and $100 \mathrm{mg}$ carbamazepine daily was prescribed. The frequency of episodes disappeared. The patient's parents (I:1 and I:2) and older sister (II:1) (Fig. 1A) did not exhibit any seizures, movement disorders or migraines. The patient's father had died in a car accident when he was 54 years old and there was no DNA available for genetic testing.

Case 2: The index patient, II:2, in Family B (Fig. 1B), a 31-year-old female, presented with paroxysmal abnormal movement since the age of 12 years. She experienced dystonia and choreoathetotic movements of the right arm; occasional clawing of the right hand; and twisted posture, grimace and impaired speech. Each episode lasted several seconds, and never exceeded $30 \mathrm{sec}$, without altered consciousness. The episodes occurred $\leq 20$ times a day. Sudden movements and acceleration were prominent triggers. Aura-like symptom of visual color enrichment usually preceded the episodes. The patient claimed that walking slowly could partially control the episodes. No positive neurological signs were identified. The results of interictal neurological examination, brain MRI and $24 \mathrm{~h}$ VEEG were normal. The patient had been prescribed $200 \mathrm{mg}$ carbamazepine daily since the age of 14 years, and a good clinical response was achieved, and was kept on $200 \mathrm{mg}$ carbamazepine every 4-5 days since. The patient's parents ( $\mathrm{I}: 1$ and $\mathrm{I}: 2$ ) were not consanguineous and the patient had two healthy siblings (II:3, 29 years old and II:4, 26 years old) and a 3 -year-old child (III:1). No neurological disorders were recorded in any other family members.

The clinical features of the two cases are summarized in Table I.

Genetics analysis. The ethical committee of the Fourth Affiliated Hospital, Zhejiang University School of Medicine approved the genetics analysis. Peripheral blood samples were collected from the patients, and their parents and siblings (Family A: I:2, II:1 and II:2; Family B: I:1, I:2, II:2, II:3 and II:4) after obtaining written informed consent from all the patients or the legal representatives of the family members. Genomic DNA was extracted using a Blood Genomic DNA Extraction kit (Qiagen, Inc.). The primers flanking all four exons and the intron-exon boundaries of PRRT2 (GenBank accession no.NM_145239) were designed using the web-based Primer 3.0 program (bioinfo.ut.ee/primer3-0.4.0/) (6-8). The PRRT2 gene was amplified by PCR. The sequences of the primers were: Exon 2-1 forward, 5'-CCCAAGCCTATCTCC TCCTC-3' and reverse, 5'-CTGGGTAGGGAGCTCTGG TT-3'; exon 2-2 forward, 5'-GACCCATGCCAAGAAACA GT-3' and reverse, 5'-GGATCCATGCAGAGAGGAGA-3'; exon 3 forward, 5'-TTCTGGGCTGGCTTCTCCT-3' and reverse, 5'-AAAGCTGCCCTTGCCAAC-3'; and exon 4 forward, 5'-CCCTGCTCTCTCCTGTCTGT-3' and reverse 5'-CTGTAAACAAGGCCGCTCAG-3'. Each PCR reaction consisted of $2 \mu \mathrm{l}$ 10X Standard Taq Reaction Buffer (Takara Bio, Inc.), $0.4 \mu 110 \mathrm{mM}$ dNTPs, $0.4 \mu \mathrm{l} 10 \mu \mathrm{M}$ forward/reverse primers, $100 \mathrm{ng}$ genomic DNA, $0.1 \mu \mathrm{l}$ Taq DNA Polymerase (Takara Bio, Inc.) and $15.7 \mu 1$ nuclease-free water. The reactions were performed in thermocyclers (PerkinElmer, Inc.), starting with an initial denaturation of 3 min at $94^{\circ} \mathrm{C}$; followed by 30 cycles of $30 \mathrm{sec}$ denaturation at $94^{\circ} \mathrm{C}, 30 \mathrm{sec}$ annealing at the primer-specific temperature, and $30 \mathrm{sec}$ extension at $72^{\circ} \mathrm{C}$. PRRT2 gene mutations were screened by direct sequencing using an ABI Prism 3100 Genetic Analyzer (Applied Biosystems; Thermo Fisher Scientific, Inc.). The sample sequences were compared with the genomic DNA sequence of PRRT2 (GenBank accession no. NM_145239).

An identical heterozygous missense mutation in both patients: c.955G $>\mathrm{T}$ (p.Val319Leu) in exon 3 of PRRT2 was detected (Fig. 1C and D). Screening of PRRT2 mutations in the patients' parents and siblings did not identify any variants. This mutation was not reported in the 1000 Genomes Project (9), and was absent from 1,200 normal controls (performed by Sanger sequencing), whose samples were collected from the Medical Examination Center, The Fourth Affiliated Hospital, Zhejiang University School of Medicine, after providing informed consent. The 1,200 normal controls consisted of 651 men and 549 women with a median age of 45 years (range, $19-78$ years). The SIFT web server (10) prediction score of the substitution was 0.024 suggesting a damaging effect of the substitution, and the PolyPhen-2 website (11) prediction score was 0.997 suggesting a probable damaging effect. The protein structures of the wild-type and mutant PRRT2 were predicted using Phyre2 (12). The results suggest that the structure of mutant PRRT2 differs notably from the wild-type structure (Fig. 2).

\section{Discussion}

PKD is often familial with autosomal dominant inheritance, but sporadic cases of PKD have also been reported (4,13-15), which are attributed to incomplete penetrance or de novo mutations (16). In the present case report, two Chinese patients with sporadic PKD were examined and a novel heterozygous missense mutation in exon 3 of the PRRT2 gene (c.955G $>$ T) was identified. The family member II: 1 and II:2 in Family B declined to have their 3 year old daughter (III:1) undergo genetic analysis. The absence of the mutation in their family members indicates the de novo status of the mutation. This newly found variant further reinforces the importance of the PRRT2 gene in PKD.

Compared with the commonly reported clinical manifestation of PRRT2-associated PKD, the patients in the present report had several primary distinctive features: i) Sporadic occurrence; ii) onset during the teenage years; iii) presented with pure PKD; iv) typically transitory spells, lasting 3-10 sec (always <30 sec); and v) episodes involved the limbs and face unilaterally. Huang et al (17) summarized the clinical manifestations and PRRT2 mutations of 110 patients with PKD, and showed that $P R R T 2$ mutation carriers were younger at onset and experienced longer episodes compared with non-PRRT2 mutation carriers (17). Previous studies have also indicated that the majority of PRRT2 mutation-positive patients experience bilateral episodes, and tend to present with complicated PKD (5). The limited sample size in the present report is inadequate for supporting the premise that the phenotype and genotype identified are correlated. To date, the role of PRRT2 is incompletely understood. Previous studies have suggested that the varied mutations observed in the PRRT2 gene may 
Table I. Clinical characteristics of the two paroxysmal kinesigenic dyskinesia cases.

Clinicopathological characteristics

\section{Sex}

Family history

Age of onset, years

Symptoms presented

Duration of symptoms

Affected limbs

Facial involvement

Presence of aura

Triggers

Frequency of episodes

Combined neurological problem

Response to treatment
Case 1

Case 2

Female

None

13

Dystonia, choreoathetosis

$<30 \mathrm{sec}$

Right

Yes

Yes

Sudden movements, acceleration 20-30 times per day before 20 years old; 1-2 times per day after 20 years old

No

Symptom-free
A

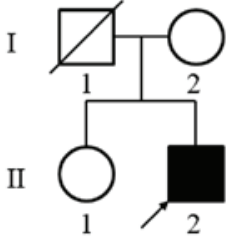

C

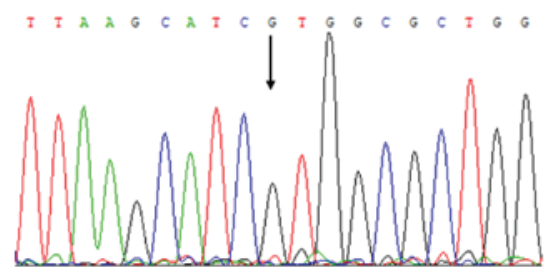

B

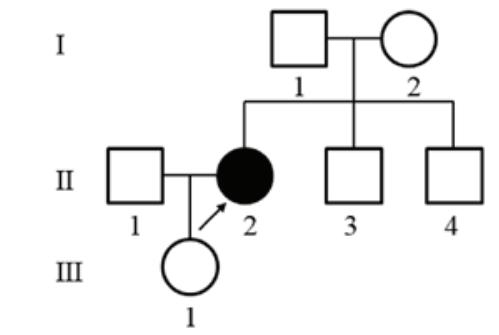

D

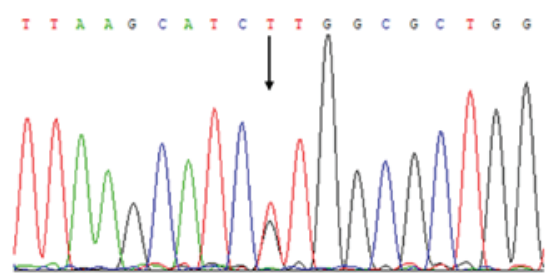

Figure 1. Family trees and sequencing analysis of the two patients with PKD. (A) Family A; patient, 18-year old male (II:2). (B) Family B; patient, 31-year old female (II:2). Arrows indicate the probands. Squares, males; circles, females; filled symbols, PKD patients; a bar across the symbol indicates a deceased individual. Sequencing analysis of the PRRT2 gene showing (C) the normal control sequence of the PRRT2 gene and (D) the c.955G $>$ T mutation. The arrow indicates the affected nucleotide. PKD, paroxysmal kinesigenic dyskinesia; $P R R 2$, proline-rich transmembrane protein 2.
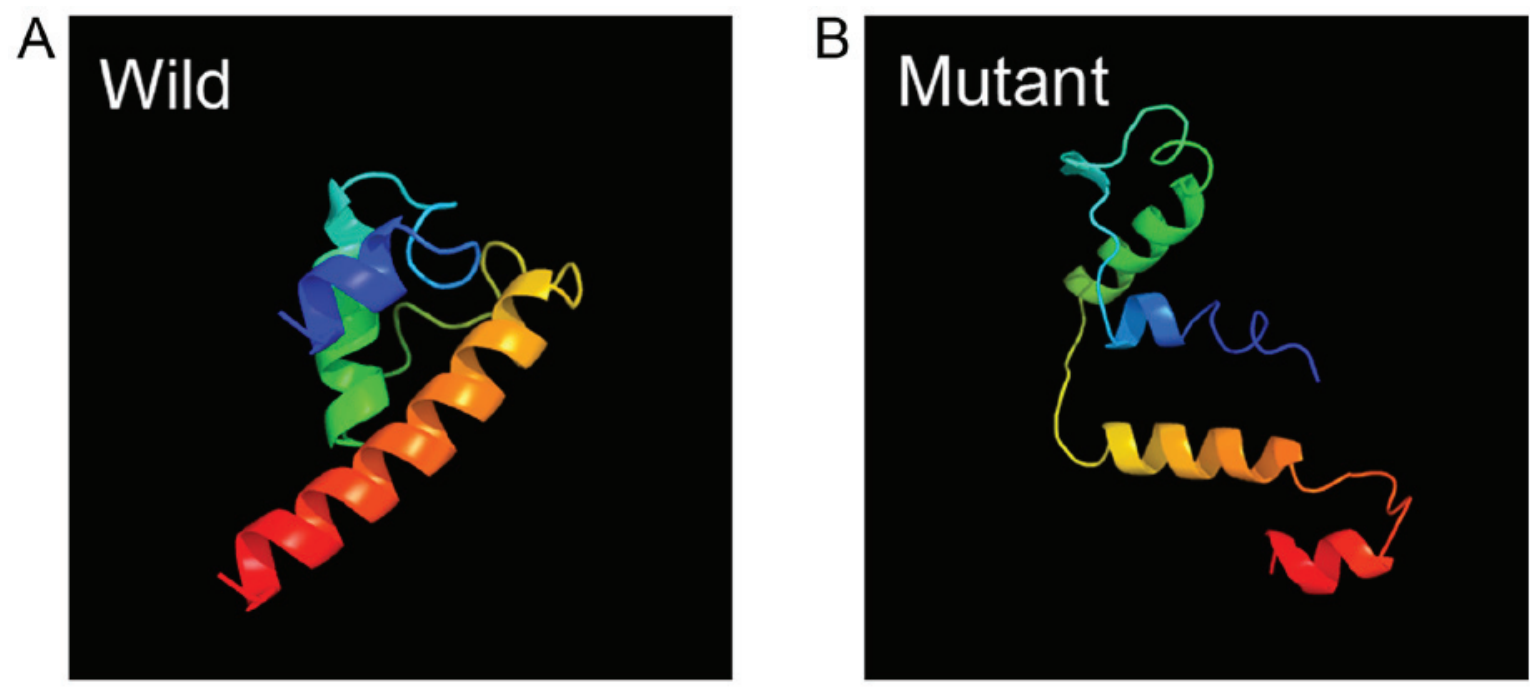

Figure 2. Predicted structures of the (A) wild-type and (B) mutant proline-rich transmembrane protein 2. 
be involved in different pathogenic molecular pathways $(4,10)$. Thus, the function of PRRT2 and its role in PKD requires further investigation.

In summary, a novel heterozygous missense mutation, c.955G $>$ T (p.Val319Leu) in exon 3 of the PRRT2 gene, was discovered in two cases of sporadic PKD. This finding expands upon the known mutation spectrum of the PRRT2 gene and may provide an opportunity for further study of the genetic pathogenesis of PKD.

\section{Acknowledgements}

Not applicable.

\section{Funding}

This work was supported by the fund of Zhejiang Province Medical Science and Technology Project (grant nos. 2017174708 and 2020RC061) and the Natural Scientific Foundation of Zhejiang Province (grant no. LGF20H090011).

\section{Availability of data and materials}

The datasets used or analyzed in the present study are available from the corresponding author on reasonable request.

\section{Authors' contributions}

JF, SW, GZ and LC prepared the experiments and wrote the manuscript. GZ performed the genetics analysis. JF, GZ and LC performed the clinical diagnoses. All authors have read and approved the final manuscript.

\section{Ethics approval and consent to participate}

Written informed consent from all the patients or the legal representatives of the family members was obtained. Informed consent was also obtained from all 1,200 healthy controls. The Ethics Committee of the Fourth Affiliated Hospital, Zhejiang University School of Medicine approved the genetics analysis.

\section{Patient consent for publication}

Written informed consent for publication was obtained from the family members.

\section{Competing interests}

The authors declare that they have no competing interests.

\section{References}

1. Erro R and Bhatia KP: Unravelling of the paroxysmal dyskinesias. J Neurol Neurosurg Psychiatry 90: 227-234, 2019.
2. Chen WJ, Lin Y, Xiong ZQ, Wei W, Ni W, Tan GH, Guo SL, He J, Chen YF, Zhang QJ, et al: Exome sequencing identifies truncating mutations in PRRT2 that cause paroxysmal kinesigenic dyskinesia. Nat Genet 43: 1252-1255, 2011.

3. Hedera P, Xiao J, Puschmann A, Momčilović D, Wu SW and LeDoux MS: Novel PRRT2 mutation in an African-American family with paroxysmal kinesigenic dyskinesia. BMC Neurol 12: 93, 2012.

4. Zhao G, Liu X, Zhang Q and Wang K: PRRT2 mutations in a cohort of Chinese families with paroxysmal kinesigenic dyskinesia and genotype-phenotype correlation reanalysis in literatures. Int J Neurosci 128: 751-760, 2018.

5. Ebrahimi-Fakhari D, Saffari A, Westenberger A and Klein C: The evolving spectrum of PRRT2-associated paroxysmal diseases. Brain 138: 3476-3495, 2015.

6. Koressaar T and Remm M: Enhancements and modifications of primer design program Primer3. Bioinformatics 23: 1289-1291, 2007.

7. Untergasser A, Cutcutache I, Koressaar T, Ye J, Faircloth BC, Remm M and Rozen SG: Primer3 - new capabilities and interfaces. Nucleic Acids Res 40: e115, 2012.

8. Kõressaar T, Lepamets M, Kaplinski L, Raime K, Andreson R and Remm M: Primer3 masker: Integrating masking of template sequence with primer design software. Bioinformatics 34 : 1937-1938, 2018.

9. 1000 Genomes Project Consortium, Auton A, Brooks LD, Durbin RM, Garrison EP, Kang HM, Korbel JO, Marchini JL, McCarthy S, McVean GA, et al: A global reference for human genetic variation. Nature 526: 68-74, 2015.

10. Sim NL, Kumar P, Hu J, Henikoff S, Schneider G and Ng PC: SIFT web server: predicting effects of amino acid substitutions on proteins. Nucleic Acids Res 40 (Web Server issue): W452-457, 2012.

11. Adzhubei IA, Schmidt S, Peshkin L, Ramensky VE, Gerasimova A, Bork P, Kondrashov AS and Sunyaev SR: A method and server for predicting damaging missense mutations. Nat Methods 7: 248-249, 2010.

12. Kelley LA, Mezulis S, Yates CM, Wass MN and Sternberg MJ: The Phyre2 web portal for protein modeling, prediction and analysis. Nat Protoc 10: 845-858, 2015.

13. Scheffer IE, Grinton BE, Heron SE, Kivity S, Afawi Z, Iona X, Goldberg-Stern H, Kinali M, Andrews I, Guerrini R, et al: PRRT2 phenotypic spectrum includes sporadic and fever-related infantile seizures. Neurology 79: 2104-2108, 2012.

14. Groffen AJ, Klapwijk T, van Rootselaar AF, Groen JL and Tijssen MA: Genetic and phenotypic heterogeneity in sporadic and familial forms of paroxysmal dyskinesia. J Neurol 260: 93-99, 2013.

15. Shi CH, Sun SL, Wang JL, Liu AQ, Miao W, Avinash C, Mao X, Tang BS and Xu YM: PRRT2 gene mutations in familial and sporadic paroxysmal kinesigenic dyskinesia cases. Mov Disord 28: 1313-1314, 2013.

16. Liu XR, Wu M, He N, Meng H, Wen L, Wang JL, Zhang MP, Li WB, Mao X, Qin JM, et al: Novel PRRT2 mutations in paroxysmal dyskinesia patients with variant inheritance and phenotypes. Genes Brain Behav 12: 234-240, 2013.

17. Huang XJ, Wang T, Wang JL, Liu XL, Che XQ, Li J, Mao X, Zhang M, Bi GH, Wu L, et al: Paroxysmal kinesigenic dyskinesia: Clinical and genetic analyses of 110 patients. Neurology 85: 1546-1553, 2015. 